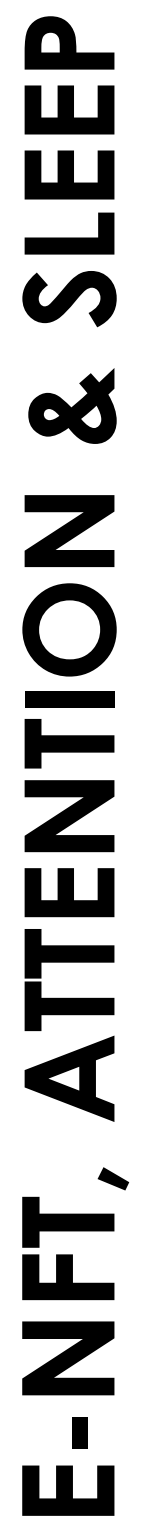

Keywords:

neurofeedback, attention, sleep, insomnia, ADHD, E-NFT

\title{
The effects of neurofeedback on attention and sleep in individuals with and without ADHD or insomnia: a literature review
}

\author{
Annel Koomen', Daniel Keeser², Sonja Verhagen ${ }^{3}$ \\ ${ }^{1}$ University of Amsterdam, Amsterdam \\ ${ }^{2}$ Ludwig Maximilian University, Munich \\ ${ }^{3}$ Hersencentrum Mental Health Institute, Amsterdam \\ *Correspondence: annel_koomen_@hotmail.com
}

DOI: $\underline{10.31739 / A N A M H .2021 .1 .30}$

\begin{abstract}
Electroencephalography-Neurofeedbacktraining (E-NFT) is a method to support subjects in learning to self-regulate their own brain activity. Besides that E-NFT may improve cognitive functions in healthy people, it may improve symptoms in different disorders, such as Attention Deficit and Hyperactivity Disorder (ADHD) and insomnia. The evidence of E-NFT for the treatment of attention problems in ADHD is still under debate, just as the suggested efficacy of E-NFT for reducing sleep problems in individuals suffering from insomnia and for improving attention and sleep in the general population. Therefore, this review examines the efficacy of E-NFT on attention and sleep in patients and healthy individuals. The reviewed literature provides evidence that standard E-NFT protocols may have a positive long-lasting effect on the inattention and hyperactivity/impulsivity symptoms in children with ADHD. In healthy children and young adults, E-NFT has been found to improve different aspects of attention and to reduce impulsivity. In addition, positive effects of E-NFT have been documented on sleep onset latency and on tiredness in healthy individuals. Sleep improvements have also been found in insomnia patients after standard ENFT, although these subjective sleep improvements may likely depend on unspecific E-NFT effects.
\end{abstract}




\section{INTRODUCTION}

Electroencephalography Neurofeedbacktraining (E-NFT), also known as electroencephalography (EEG)

biofeedback, is a method wherein subjects learn to selfregulate their own brain activity [1]. During E-NFT, the subject's brain waves are continuously measured with EEG and instant positive or negative feedback in the form of audio and/or video based on this activity is provided to the subject [2]. E-NFT is not only used to improve functions in healthy persons [3-7], but also to treat different disorders, such as attention deficit hyperactivity disorder (ADHD) and insomnia [8]. Children with ADHD have several inattentive and/or hyperactivity/impulsivity (HI) symptoms, while insomnia is characterized by difficulties with initiating and maintaining sleep and by early-morning awakenings [9]. In addition, both disorders are accompanied with impairments in daily life functioning [9]

The E-NFT protocols used for the treatment of ADHD and/or insomnia include amongst others theta/beta ratio (TBR), slow cortical potential (SCP) and sensorimotor rhythm (SMR) E-NFT [10]. These E-NFT protocols are well-studied in children with ADHD and therefore considered to be standard E-NFT protocols [11]. TBR ENFT is aimed to decrease the TBR; thus to increase the power of beta waves over theta waves [10], which is found to be decreased in children and adolescents with ADHD $[12,13]$. Notably, beta waves are found when a person is alert, and theta waves when a person is sleepy [8]. Furthermore, SCP E-NFT is used to improve the direction of SCPs [8], which are associated with increased attention [14, 15]. It has been shown that 36 sessions of combined TBR and SCP E-NFT in children with ADHD decreased inattentive, HI and total ADHD symptoms when compared to children with ADHD after computerized attention skills training sessions [16]. Furthermore, children with ADHD showed significant improvements in inattentive symptoms after receiving 30 sessions of SCP E-NFT as compared to the children with ADHD that had underwent group sessions [17]. The overall ADHD symptoms were also improved after 25 SCP neurofeedback sessions in children with ADHD as compared to children with ADHD that were on a waiting list (Heinrich et al., 2004). Improved scores on a sustained attention task after 20 sessions beta power enhancing $\mathbf{E}$ NFT (short: beta E-NFT) was found in healthy subjects as well, but only when the beta waves were changed after ENFT [18].

Another E-NFT protocol that is aimed to enhance the SMR power across sensorimotor areas called SMR E-NFT [8]. The sensorimotor area is a cortical area that integrates sensory and motor information [19]. Improved inattention and impulsivity symptoms were found in children with
ADHD after 36 sessions of SMR as well as TBR E-NFT [20]. SMR E-NFT in the treatment of children with ADHD started because of its beneficial effect on motor inhibition [21, 22]. In addition, in a young epileptic patient sleep onset latency was reduced after 12 sessions of SMR E-NFT [23]. Many years later, the effects of E-NFT were studied in people with psychophysiological insomnia, which is a type of insomnia caused by psychological and physiological factors such as tension [24]. No sleep improvements were found after sessions of SMR E-NFT, electromyographic (EMG) biofeedback or combined EMG biofeedback and theta power enhancing E-NFT (short: theta E-NFT) as compared to no feedback [25]. EMG biofeedback is, in contrast to E-NFT, not based on the subject's brain waves but on their muscular tension, with the aim to reduce this tension. The amount of SMR enhanced during SMR E-NFT, however, correlated positively with sleep improvements in this study. Also, baseline tension was correlated negatively with sleep improvements for the SMR E-NFT group, and positively with sleep improvements for the EMG biofeedback group. In a follow-up study people with psychophysiological insomnia showed increased total time of sleep per night and reduced sleep onset latency after six sessions of EMG biofeedback and 26 sessions of either theta or SMR ENFT [26]. The number of awakenings were decreased after theta E-NFT as well. As in line with the previous study, baseline tension of the insomnia patients correlated positively with sleep improvements after theta E-NFT, and negatively with the sleep improvements after SMR E-NFT. Because in the early 80's the number of sessions needed to increase desired EEG power was high and E-NFT equipment was expensive, studies examining E-NFT effects were no longer performed [26]. Due to technological advantages, studies using E-NFT interventions were continued decennia later [27]. One such technological advantage is the introduction of E-NFT based on Z-scores, which is the deviation of the subjects EEG measured at multiple sites as compared to a normative EEG database of healthy people [28]. Case studies in people with various disorders such as autism spectrum disorder indicated improved sleep pattern after Z-score based E-NFT [28]. However, recent studies should be evaluated to more substantially clarify the efficacy of E-NFT for improving sleep and decreasing sleep problems.

The efficacy of E-NFT in ADHD has been investigated in a meta-analysis with controlled studies investigating E-NFT and its effect in children with ADHD [29]. In this study, large effect sizes were found for impulsivity and inattention and a medium effect size for hyperactivity, therefore stating E-NFT as an efficacious and specific treatment for ADHD. However, a lot of these controlled studies showed important methodological problems [30], such as 
the lack of a specific control group [31, 32] or missing the randomization of group assignment [20, 31]. The evidence of E-NFT for the treatment of ADHD was therefore recently considered insufficient [33]. A general effect of ENFT on attention, in healthy people as well, is also not substantialized.

In summary, the clinical efficacy of E-NFT in the treatment of ADHD and insomnia is not robust and undebatable. This is also the case for the effects of E-NFT on attention and sleep in healthy subjects. Therefore, the aim of this literature review is to further examine the efficacy of EEG-NFT on attention and sleep in individuals with ADHD or sleeping problems and in healthy individuals. First, the E-NFT effects on ADHD symptoms in children are discussed. Second, the E-NFT effects on attention in healthy children and young adults are examined. Third, the E-NFT effects on sleep in insomnia patients are discussed. Lastly, the E-NFT effects on sleep in healthy individuals are reviewed.

\section{Neurofeedback effects on ADHD symptoms in children: results from meta-analyses of randomized controlled trials}

In this section, the E-NFT effects on ADHD symptoms in children with ADHD are discussed based on the results of meta-analyses of randomized controlled trials (RCTs). In these RCTs, the ADHD symptoms and subscales of inattention and $\mathrm{HI}$ are measured before and after the experimental E-NFT or control condition. With respect to the control condition studies with medication interventions as a control condition are excluded. The change in symptoms after relative to before E-NFT is used to calculate the E-NFT effects. In the case of children as participants, symptoms are rated by someone other than the subject itself, most often the children's parents and/or teachers [34]. In the meta-analyses, a distinction between probably blinded (PB) and probably unblinded (PU) raters are made. $\mathrm{PB}$ raters, often the participant's teachers [10], are likely unaware of the participant's condition because they are not that much invested in the E-NFT, or are unaware of the participant's condition when it concerns a placebo-controlled RCT [35]. In contrast, PU raters are normally the participant's parents [10]. They are closest to the therapeutic setting and therefore often aware of the participant's condition [35]. The E-NFT effects in children with ADHD on ADHD symptoms, based on PU and $\mathrm{PB}$ raters, of all meta-analyses are summarized in Table 1 .

In 2016, a meta-analysis of 13 RCTs was performed by Cortese et al. in children with ADHD that received ENFT as experimental condition. A small-to-moderate but significant improvement on inattention, HI, and total ADHD symptoms after E-NFT was found as compared to the control condition when the scores of the PU raters were used. When considering the PB assessments, all improvements were no longer significant. An additional analysis of RCTs that met the standard E-NFT protocol criteria set up by Arns et al. [36] ( $n=7)$, also found a significant decrease in inattention, HI, and total ADHD symptoms rated by PU assessors. The inattentive and total ADHD symptoms were found to be decreased as well by the RCTs with both standard E-NFT protocols and PB assessments $(n=3)$, while the hyperactivity symptoms were not changed. These improvements were also found, notably with larger effect sizes, after replicating and updating this meta-analysis by including recent RCTs $(n=3)[37]$.

These results are in line with the findings of a recent metaanalysis of RCTs using a standard protocol E-NFT for the experimental condition in children with ADHD [38]. When the RCTs using PU assessments were analyzed $(n=11)$, a decrease in inattentive and HI symptoms was found after E-NFT as compared to the control condition. When the RCTs with PB assessments were used ( $n=9)$, a significant decrease of inattentive symptoms, but no significant difference in HI symptoms was found. The total ADHD symptoms were not investigated in this study.

In the above cited meta-analysis of [39], a separate analysis was performed in studies using active and placebo control groups. In addition, studies with control conditions resembling treatment as usual and waiting list periods were excluded. The remaining RCTs had control conditions including cognitive training, EMG biofeedback and placebo-controlled E-NFT. With respect to PU assessments, E-NFT was found to improve the HI symptoms in the children with ADHD, with a small effect size, but the inattention and total ADHD symptoms were not different. Moreover, the inattention, HI, and total ADHD symptoms were not different when only the PB assessments were used.

Another meta-analysis showed different results when using 5 RCTs with the same control conditions as described in the meta-analysis above [40]. When considering the PU assessments, improvements were found on inattention, HI, and total ADHD symptoms after E-NFT as compared to the control condition. When considering the PB assessments, the inattention symptoms, but not $\mathrm{HI}$ and total ADHD symptoms, were found to improve. This is in contrast with the findings of Cortese et al. [39], who only found an improvement in HI symptoms for the PU measurements. This finding can be explained by the exclusion of nonstandard E-NFT procedures and measures of only PU measurements by MicoulaudFranchi et al. [40], which was not the case in the study of Cortese et al. [39]. In addition, larger effect sizes for all 
outcomes and smaller effect sizes for the PU outcomes were found in the study of Micoulaud-Franchi et al. [40] as compared to Cortese et al. [39]. Furthermore, the results of Micoulaud-Franchi et al. [40] are in line with the results of Riesco-Matías et al. [38], who also found significant improvements in ADHD symptoms for the PU measurements, and in inattentive scores for the PB measurements.

A quite recent meta-analysis included 10 RCTs that also examined long-term effects of E-NFT on the ADHD symptoms in children with ADHD [41]. The PU measurements were used as the outcomes. A significant improvement was found for both inattention and HI symptoms after the neurofeedback sessions, as well as after 2 to 12 months following the E-NFT, as compared to the control conditions. Interestingly, the effect sizes were increased at follow-up as compared to post E-NFT for both subscales. When the E-NFT group was analyzed apart from the control group, a medium effect size of $\mathrm{E}$ NFT was found after treatment, and a large effect size of E-NFT at follow-up was found for inattention symptoms. For HI symptoms, medium effect sizes were found after E-NFT and at follow-up. The results were the same when only standard E-NFTs were considered.
A meta-analysis of studies on E-NFT efficacy in children with ADHD, examined the experimental and clinical factors influencing this efficacy [37]. For this purpose, also non-controlled and non-randomized studies were included. A total of 33 studies were analyzed. The E-NFT effect on ADHD symptomatology was found to be most dependent of the amount of blindness of the raters, the total treatment length and EEG quality. In addition, ENFT efficacy was higher when the EEG quality was higher, and when the total treatment length was shorter. The assessment of a PB rater did reduce the efficacy. Importantly, they found that this reduced efficacy was probably due to the notion that blinded raters scored the ADHD symptoms significantly lower before E-NFTs at baseline, instead of significantly higher after E-NFT, as compared to PU raters. Also the influence of amongst others ADHD severity at baseline, control group presence and the type of reward in the E-NFT protocol on the ENFT efficacy in children with ADHD could not be examined because of too many missing and too heterogenous data points. The type of E-NFT protocol used, TBR E-NFT, SMR E-NFT or SCP E-NFT, did not seem to differentiate the E-NFT outcome in children with ADHD. 
Table 1. Results of the meta-analyses of RCTs on the effect of E-NFT in children with ADHD on ADHD symptoms based on PU and PB raters.

\begin{tabular}{|c|c|c|c|}
\hline Meta-analysis & Studies analyzed & $\begin{array}{l}\text { PU rater based effects after E-NFT } \\
\text { (number studies analyzed) }\end{array}$ & $\begin{array}{l}\text { PB rater based effects } \\
\text { after E-NFT (number of } \\
\text { studies analyzed) }\end{array}$ \\
\hline \multirow[t]{3}{*}{$\begin{array}{l}\text { Cortese et al. } \\
\text { (2016 [39]) }\end{array}$} & All RCTs & $\begin{array}{l}\text { Inattention symptoms improved }(n=11) \\
\text { HI symptoms improved }(n=10) \\
\text { Total ADHD symptoms improved }(n=13)\end{array}$ & $\begin{array}{l}\text { Inattention symptoms } \\
\text { unchanged }(n=7) \\
\text { HI symptoms unchanged } \\
(n=7) \\
\text { Total ADHD symptoms } \\
\text { unchanged }(n=8)\end{array}$ \\
\hline & $\begin{array}{l}\text { Standard E-NFT } \\
\text { protocol RCTs }\end{array}$ & $\begin{array}{l}\text { Inattention symptoms improved }(n=5) \\
\text { HI symptoms improved }(n=5) \\
\text { Total ADHD symptoms improved }(n=7)\end{array}$ & $\begin{array}{l}\text { Inattention symptoms } \\
\text { improved ( } n=3) \\
\text { HI symptoms unchanged } \\
(n=3) \\
\text { Total ADHD symptoms } \\
\text { improved }(n=3)\end{array}$ \\
\hline & $\begin{array}{l}\text { RCTs with active } \\
\text { and placebo } \\
\text { control groups }\end{array}$ & $\begin{array}{l}\text { Inattention symptoms unchanged }(n=6) \\
\text { HI symptoms improved }(n=6) \\
\text { Total ADHD symptoms unchanged }(n=7)\end{array}$ & $\begin{array}{l}\text { Inattention symptoms } \\
\text { unchanged }(n=5) \\
\text { HI symptoms unchanged } \\
(n=5) \\
\text { Total ADHD symptoms } \\
\text { unchanged }(n=6)\end{array}$ \\
\hline \multirow[t]{2}{*}{$\begin{array}{l}\text { Bussalb et al. } \\
\text { (2019 [37]) }\end{array}$} & All RCTs & $\begin{array}{l}\text { Inattention symptoms improved }(n=13) \\
\text { HI symptoms improved }(n=12) \\
\text { Total ADHD symptoms improved }(n=16)\end{array}$ & $\begin{array}{l}\text { Inattention symptoms } \\
\text { unchanged }(n=8) \\
\text { HI symptoms unchanged } \\
(n=8) \\
\text { Total ADHD symptoms } \\
\text { unchanged }(n=9)\end{array}$ \\
\hline & $\begin{array}{l}\text { Standard E-NFT } \\
\text { protocol RCTs }\end{array}$ & $\begin{array}{l}\text { Inattention symptoms improved }(n=6) \\
\text { HI symptoms improved }(n=6) \\
\text { Total ADHD symptoms improved }(n=9)\end{array}$ & $\begin{array}{l}\text { Inattention symptoms } \\
\text { improved }(n=4) \\
\text { HI symptoms unaffected } \\
(n=4) \\
\text { Total ADHD symptoms } \\
\text { improved }(n=4)\end{array}$ \\
\hline $\begin{array}{l}\text { Riesco-Matías et } \\
\text { al. (2019 [38]) }\end{array}$ & $\begin{array}{l}\text { Standard E-NFT } \\
\text { protocol RCTs }\end{array}$ & $\begin{array}{l}\text { Inattention symptoms improved }(n=11) \\
\text { HI symptoms improved }(n=11)\end{array}$ & $\begin{array}{l}\text { Inattention symptoms } \\
\text { improved }(n=9) \\
\text { HI symptoms unchanged } \\
(n=9)\end{array}$ \\
\hline $\begin{array}{l}\text { Micoulaud- } \\
\text { Franchi et al. } \\
\text { (2014 [40]) }\end{array}$ & $\begin{array}{l}\text { Standard E-NFT } \\
\text { protocol RCTs } \\
\text { with active and } \\
\text { placebo control } \\
\text { groups }\end{array}$ & $\begin{array}{l}\text { Inattention symptoms improved }(n=5) \\
\text { HI symptoms improved }(n=5) \\
\text { Total ADHD symptoms improved }(n=5)\end{array}$ & $\begin{array}{l}\text { Inattention symptoms } \\
\text { improved }(n=5) \\
\text { HI symptoms unchanged } \\
(n=5) \\
\text { Total ADHD symptoms } \\
\text { unchanged }(n=5)\end{array}$ \\
\hline $\begin{array}{l}\text { van Doren et al. } \\
\text { (2019 [41]) }\end{array}$ & $\begin{array}{l}\text { RCTs with two to } \\
12 \text { months follow- } \\
\text { up data }\end{array}$ & $\begin{array}{l}\text { Inattention symptoms improved }(\mathrm{n}=10) \\
\mathrm{HI} \text { symptoms improved }(\mathrm{n}=10) \\
\text { After } E-N F T \text {, as well as follow-up }\end{array}$ & \\
\hline
\end{tabular}

Note: ADHD = attention deficit hyperactivity disorder, HI = hyperactivity/impulsivity, E-NFT $=$ EEG neurofeedbacktraining, $\mathrm{PB}=$ probably blinded, $\mathrm{PU}=$ probably unblinded, $\mathrm{RCT}$ = randomized controlled trial 
The finding that the type of E-NFT protocol had no significant effect on the ADHD symptoms in children with ADHD was also in line with two other studies that compared E-NFT protocols. In the first study, children with ADHD were randomly assigned to a group receiving 30 sessions TBR E-NFT $(n=19)$ or 30 sessions SCP ENFT $(n=19)$ [42]. In another study, 37 ADHD patients were assigned to a group receiving TBR E-NFT $(n=10)$ or SMR E-NFT ( $\mathrm{n}=27$ ), for on average 29 and 31 sessions respectively [11]. In both studies significant improvements were found in inattention and HI symptoms over the course of the sessions. This effect was not significantly different between the E-NFT protocol, so not significant different between the TBR as compared to the SCP protocol and between the TBR as compared to the SMR protocol. This was true for both the PB and PU measures.

\section{CONCLUSION I}

The meta-analyses provide evidence that E-NFT treatment has a positive effect on inattention and HI symptoms in children with ADHD. This effect seems to last over a longer period of time, with even a better improvement for the inattention symptoms after the ending of the $\mathrm{E}$ NFT treatment. This effect is more robust when standard E-NFT protocols are used, while the type of standard E-NFT protocol seems not to differently affect the outcomes. The improvement in inattentive symptoms are found when the PB as well as the PU measures are used. The HI symptoms are found to be improved when the PU measures are used but not the PB. However, it is thought that this difference is caused by an underestimation of these symptoms by $\mathrm{PB}$ raters. In addition, $\mathrm{PB}$ raters rate these symptoms already low before the E-NFT treatment, as compared to the more proximal PU raters, therefore making improvement less possible. As a consequence, it is thought that E-NFT treatment has a positive effect on both the HI and inattention symptoms.

\section{Neurofeedback effects on attention in healthy children and young adults}

In this section, the E-NFT effects on attention and impulsivity are reviewed in children and young adults without ADHD. Different aspects of attention are measured in the studies, which are sustained attention, selective attention and attention control. Sustained attention is the capacity to maintain focus and alertness over time [43]. The focus on a specific target for enhanced processing is called selective attention [43]. Lastly, attention control is the ability to regulate responses, especially in conflict situations [44]. It must be noted that sustained attention, selective attention and attentional control overlap and often more attentional processes are needed in attentional tasks.

An RCT in children without attentional problems could not find an effect of TBR E-NFT on sustained and selective attention [45]. The $\mathrm{d} 2$ test was used to measure selective and sustained attention in 47 children of 8 and 9 years. The children were randomly assigned to an TBR ENFT $(n=24)$ and control group that did not receive E-NFT $(\mathrm{n}=23)$. The $\mathrm{d} 2$ test was performed before and after one week, wherein the TBR E-NFT group received 10 sessions of TBR E-NFT. Children with scores on the $\mathrm{d} 2$ test indicative for ADHD were excluded from the study. After this week, a significant improvement in processing speed, accuracy of processing and total effectiveness on the test was found, but this was found in both the TBR ENFT and control condition and appeared not to be different between the groups, although effect sizes in the experimental group were larger than in the control group.

When different attention tasks were used, a positive effect of TBR E-NFT on selective attention was found in healthy children [46]. Twenty-nine children of 10-13 years old were divided in a TBR E-NFT group $(n=12)$ and sham $\mathrm{E}$ NFT group ( $\mathrm{n}=15)$, wherein EEG was measured but no feedback was provided. Selective attention was measured before and after ten to 15 neurofeedback sessions in two weeks. TBR was significantly decreased within the neurofeedback sessions in the TBR E-NFT and not sham E-NFT group. Furthermore, different aspects of selective attention which were working efficiency measured with Schulte's tables and productivity of attention measured with Bourdon's correcture test was improved in the experimental group only.

In healthy young adults, there seems to be a positive effect on sustained attention after SMR and TBR E-NFT, reflected by better performance after SMR E-NFT and faster responses after TBR E-NFT [47]. In this RCT, 25 students of $21 \pm 2.24$ (SD) year without mental or neurological illness not using medication were randomly assigned to a SMR E-NFT ( $\mathrm{n}=9)$, beta E-NFT $(\mathrm{n}=8)$ and behavioral training control group $(\mathrm{n}=8)$. Additionally, theta and high beta power were reduced during SMR and beta E-NFT as well. Notably, theta waves are associated with being sleepy and high beta waves with being hyperalert and anxious [8]. Sustained attention was measured with a continuous performance task (CPT) and divided attention task before and after ten sessions in ten weeks. While 
baseline measures on the tasks were not different between the groups before E-NFT, significant faster reaction times on the CPT were found after beta E-NFT as compared to the control group. Furthermore, results on the divided attention task showed that omission errors were reduced and hit to false rate ratios were increased after SMR neurofeedback sessions as compared to the control group.

Another study also found a decreased number of omission errors and increased hit rates on a CPT in young adults in the SMR E-NFT group only [48]. Thus, this also suggests a positive effect of SMR E-NFT on sustained attention and better performance. In this single-blind RCT, 29 students of 20-28 year were randomly assigned to one of three groups which received SMR E-NFT $(n=10)$ while inhibiting theta and beta power, theta E-NFT in sensorimotor areas $(n=9)$ while inhibiting power of alpha and delta power, and a Non-NFT control group $(\mathrm{n}=10)$. Notably, alpha waves are associated with being relaxed, and delta waves with being unaware [8]. Sustained attention was measured with a CPT before and after 8 neurofeedback sessions in 4 weeks. No differences on the task were found after theta E-NFT. In addition, theta was not significantly increased within the sessions of theta $\mathrm{E}$ NFT, while SMR was significantly increased within the sessions of SMR E-NFT.

In a double-blind RCT using Z-based E-NFT instead of standard E-NFT protocols, no effect of E-NFT on sustained attention and impulsivity was found in young adults (Logemann et al., 2010). Twenty-seven healthy students that scored relatively high on impulsivity and inattention questionnaires were randomly assigned to individualized protocols based on Z-scores $(\mathrm{n}=14)$ and sham E-NFT group $(\mathrm{n}=13)$. The sham E-NFT group received E-NFT based on a simulated EEG signal. No significant differences were found after 16 neurofeedback sessions in eight weeks on the reaction time on a CPT measuring sustained attention. This was also the case for the reaction time and false-alarm rate on CPTs related to impulsivity.

In a single-blind RCT, school children aged 11 years were randomly assigned to 10 sessions of SMR E-NFT ( $\mathrm{n}=9$ ), 10 sessions of theta alpha ratio (TAR) E-NFT $(n=10)$ or to a non-treatment control group $(\mathrm{n}=11)$ [49]. During TAR, an increase in power of theta waves over alpha waves and thus an increase in TAR is aimed [50]. Alpha waves are associated with being relaxed and with being alert, and theta waves with being sleepy [8]. For SMR E-NFT, it was also aimed to reduce theta and high beta power. Within the sessions, significant excess SMR over high beta power in the SMR E-NFT group and increased TAR in the TAR E-NFT group was found. Across neurofeedback sessions, no significant differences in EEG power was found.
Commission errors in a CPT, reflecting impulsivity, was significantly reduced after TAR as compared to the control group. No differences were found on the CPT after SMR E-NFT. This was in line with the results of Egner \& Gruzelier [47], who used the same task and also did not find significant differences after SMR. Thus, TAR E-NFT seems to have a positive effect on impulsivity in children.

A positive effect of theta E-NFT in frontomedial brain areas on attention control was found in heathy young adults [51]. In this single-blind RCT, healthy young adults of 21-25 year old who were free from neurological, psychological and mood disorders were randomly assigned to a theta E-NFT condition $(\mathrm{n}=8)$ and sham $\mathrm{E}$ NFT control condition $(\mathrm{n}=8)$. Sham E-NFT was aimed to enhance random bins in frequencies ranging from alpha to high beta waves. An attention network task was performed before and after 12 neurofeedback sessions in four weeks. Theta power, as well as executive attention or attention control, was enhanced after the 12 theta neurofeedback sessions as compared to the 12 sham neurofeedback sessions.

\section{CONCLUSION II}

The majority of findings provide evidence of positive effects of standard E-NFT protocols on attention and impulsivity in children and young adults without ADHD. In addition, sustained and selective attention might be improved by TBR E-NFT in these children and young adults. SMR E-NFT seems to have a positive effect on sustained attention in these young adults. More specifically, better performance after SMR ENFT and faster responses after TBR E-NFT were found. Impulsivity might be improved by TAR E-NFT in children, and attention control after theta E-NFT in young adults.

\section{Neurofeedback effects on sleep in insomnia patients}

In this section, studies examining the E-NFT effects on sleep in insomnia patients are discussed, with a focus on SMR E-NFT. As can be seen in the preceding sections as well, SMR E-NFT is often accompanied with the reduction of high beta and theta power at these areas as well. In addition, high beta waves are associated with hyperalertness and anxiety $[8,52]$. Theta waves are 
associated with being sleepy, and only delta waves are lower in frequency, which are associated with being unaware and asleep [8]. Furthermore, to measure sleep polysomnography (PSG) during sleep or questionnaires are used in these studies. These measurements are done before and after the experimental or control condition. Also, sleep diaries that are kept by the subjects throughout the experiment are used to calculate specific sleep parameters. The change in these sleep variables in insomnia patients after as compared to before E-NFTs are discussed below. The results of the studies in this section are summarized in Table 2.

In a single-blind RCT, 17 insomnia patients were randomly assigned to SMR E-NFT $(n=9)$ and EMG biofeedback control group $(n=8)$ [2]. Medication-free patients with primary insomnia symptoms, assessed with questionnaires and a semi-structured psychiatric interview, for a minimum of three times a week for at least six months were included. Besides increasing SMR power, ENFT was administered to reduce theta and high beta power in sensorimotor areas. Twenty E-NFT or EMG biofeedback sessions were performed at home with a portable device. As a consequence, potential biases of informed clinicians during E-NFT were excluded as well, which is also the case for double-blind studies. Sleep was measured objectively using PSG and subjectively using sleep diaries. Insomnia patients without objective sleep problems were excluded from the study. The baseline somatic arousal and baseline tension level was not higher in the insomnia patients of each group as compared to a group of 12 healthy individuals. In the E-NFT relative to the control group, an increase in objective total sleep time was observed, as well as an improvement in subjective sleep variables, which were the total sleep time, wake after sleep onset and sleep onset latency. In both the E-NFT and biofeedback group, the sessions shortened the objective wake after sleep onset and sleep onset latency and increased the total rapid eye movement (REM) sleep and subjective sleep efficiency. There was no association between the baseline EMG tension level and sleep outcome after each feedback session found in the insomnia patients. This suggests that E-NFT aimed to enhance SMR and to reduce theta and high beta power in sensorimotor areas and EMG biofeedback both have a positive effect on the objective sleep onset latency, wake after sleep onset, amount of REM sleep and subjective sleep efficiency in insomnia patients. Furthermore, objective and subjective total sleep time and subjective sleep onset latency and wake after sleep onset was found only after SMR E-NFT.

An improvement in subjective total sleep time, insomnia severity and sleep quality was observed in insomnia patients after Z-score based E-NFT protocols [27]. In this single-blind pilot-study, eight medication-free patients with a Diagnostic and Statistical Manual of Mental Disorders (DSM) IV primary insomnia diagnosis and poor subjective sleep quality based on a questionnaire were randomly assigned to an individualized E-NFT protocol $(n=5)$ or SMR E-NFT protocol that was also aimed to reduce theta and high beta power at sensorimotor areas $(n=3)$. Z-scores were used to continuously provide feedback and to normalize amplitude and connectivity at variables at multiple sites for the individualized E-NFT protocol and at sensorimotor areas for the SMR E-NFT protocol. Besides sleep variables measured with sleep diaries, questionnaires were administered as well. Based on the data of these questionnaires, the sleep quality, sleep efficiency, insomnia severity and quality of life was improved after E-NFT as well. All these improvements were found when using the data of both conditions, and thus independent of E-NFT protocol. After E-NFT, the beta and delta waves were significantly normalized, and a trend towards normalized high beta waves was also found. It must be noted, however, that in this study a small sample and no control group was present.

A subsequent RCT in 40 insomnia patients was therefore performed, using the same Z-based SMR E-NFT protocol as Hammer et al. [27] as experimental E-NFT condition [53]. Patients that met the DSM-5 criteria for insomnia disorder, had poor subjective sleep quality and did not take medication for their sleep complaints were included. These participants were randomly assigned to a group receiving 20 sessions of SMR E-NFT $(n=10)$, nine sessions of cognitive behavioral therapy (CBT) $(n=10)$ or no treatment as a control group $(n=20)$. In this study, significant enhanced SMR and reduced high beta power in sensorimotor areas was found within the neurofeedback sessions across the sessions. Sleep quality and insomnia severity was measured with questionnaires before and after finishing the treatments in three months. Both SMR ENFT and CBT improved the subjective sleep quality, as compared to the control condition. This improvement related to the control group was significantly higher for the SMR E-NFT group as compared to the CBT group. Furthermore, a significant reduction in insomnia severity was found after E-NFT as compared to the CBT and control condition. Thus, SMR E-NFT has a positive effect on insomnia severity and sleep quality in insomnia patients, and was even more effective than common treatments for insomnia. The control group in this study, however, did not receive any treatment and thus did not control for any unspecific effects such as the participant's expectation.

Another single-blind RCT used sham E-NFT as a placebo-controlled condition in insomnia patients [54]. During sham E-NFT, five random frequency bands in the 
beta range except for SMR frequencies were used as rewarded frequency bands. The authors found that successful SMR but also unwanted high beta E-NFT in sensorimotor areas improved the objective amount of awakenings and slow wave sleep (SWS) and possibly the subjective sleep quality in insomnia patients. In addition, 24 participants with primary insomnia received ten SMR neurofeedback sessions as well as five sham neurofeedback sessions in a counterbalanced manner. The research diagnostic criteria for primary insomnia [55], with insomnia symptoms occurring at least three times a week, and the DSM-III-R criteria for sleep disorders was used to determine primary insomnia. Poor subjective sleep quality based on sleep diaries and abstinence of medication throughout the experiment was also needed. Sleep was measured using PSG, sleep diaries and questionnaires. In the sensorimotor areas, SMR power was significantly enhanced after SMR E-NFT as compared to sham E-NFT, as well as high beta power. After the SMR E-NFT as compared to sham neurofeedback sessions, improvements in PSG measurements were found. These improvements were a reduction of number of awakenings and an increase of SWS. No significant difference in other PSG measures were found such as the total sleep time, waking after sleep onset, sleep onset latency, sleep efficiency, time in bed and amount of sleep stages other than SWS. These were also not found for sleep measured based on the sleep diaries of the subjects, including the total sleep time, sleep onset latency and sleep efficiency. Furthermore, the physical quality of life, assessing domains such as energy and fatigue, and sleep quality, both measured with questionnaires, was significantly improved after E-NFT. However, for the physical quality of life this effect was found for both SMR and sham E-NFT, while it was not studied whether improvement in subjective sleep quality was also independent of E-NFT protocol. Furthermore, more social support was experienced by the subjects when they were in the experimental as compared to the control condition

The previous study was therefore replicated in 25 medication-free patients that met the research criteria for primary insomnia [55], but included some changes to eliminate unspecific effects such as social support [56]. First of all, clinicians were not informed of the condition of the subjects, making it a double-blind RCT. Also, 12 sessions of SMR E-NFT as well as sham E-NFT were used to increase the specificity of the control group. Furthermore, insomnia patients without objective sleep problems were excluded. No sleep improvements were found after the SMR as compared to sham neurofeedback sessions. Only a significant improvement after both SMR and sham E-NFT was found on sleep quality and physical quality of life, irrespective of type of E-NFT, which is in line with the results of Schabus et al. [54]. No difference after E-NFT was found for other sleep parameters measured with PSG and questionnaires. Also no relationship between more relaxed and less tense insomnia patients and better objective sleep quality after specifically SMR E-NFT was found. Lastly, SMR power was enhanced within the neurofeedback sessions across all 12 sessions, but was not higher after E-NFT as compared to before E-NFT as in contrast to Schabus et al. [54]. Absence of increased SMR power after SMR E-NFT was also found in healthy young adults that received the same E-NFT protocol, and is therefore probably not due to learning impairments that can be found in insomnia patients because of their disease. These results suggest that improved subjective sleep quality and physical quality of life in insomnia patients is rather due to unspecific E-NFT effects such as social support, motivation and expectation, because they are not related to the trained frequency after E-NFT. 
Table 2. Results of the studies on the effect of SMR E-NFT in insomnia patients on different sleep variables.

\begin{tabular}{|c|c|c|c|c|}
\hline Study & $\begin{array}{l}\text { Experimen } \\
\text { tal group } \\
\text { (amount } \\
\text { sessions) }\end{array}$ & $\begin{array}{l}\text { Contr } \\
\text { ol } \\
\text { group } \\
\text { (amo } \\
\text { unt } \\
\text { sessi } \\
\text { ons) }\end{array}$ & $\begin{array}{l}\text { Sleep variable (measurement } \\
\text { method) / } \\
\text { if available: EEG data }\end{array}$ & Result \\
\hline \multirow{2}{*}{$\begin{array}{l}\text { Cortoos } \\
\text { et al. } \\
(2010 \\
[2])\end{array}$} & \multirow{2}{*}{$\begin{array}{l}\text { SMR E- } \\
\text { NFT, } \\
\text { inhibiting } \\
\text { theta and } \\
\text { high beta } \\
\text { (20) }\end{array}$} & \multirow{2}{*}{$\begin{array}{l}\text { EMG } \\
\text { biofe } \\
\text { edbac } \\
\text { k (20) }\end{array}$} & $\begin{array}{l}\text { Total sleep time }(\mathrm{PSG}+\mathrm{S}) \\
\text { Sleep onset latency (S) } \\
\text { Wake after sleep onset (S) }\end{array}$ & $\begin{array}{l}\text { Higher improvement after sessions for } \\
\text { experimental as compared to control group }\end{array}$ \\
\hline & & & $\begin{array}{l}\text { Sleep onset latency (PSG) } \\
\text { Wake after sleep onset (PSG) } \\
\text { REM sleep (PSG) } \\
\text { Sleep efficiency (S) }\end{array}$ & $\begin{array}{l}\text { Improved after sessions, for both the } \\
\text { experimental and control group }\end{array}$ \\
\hline \multirow{3}{*}{$\begin{array}{l}\text { Hammer } \\
\text { et al. } \\
\text { (2011 } \\
[27])\end{array}$} & \multirow{3}{*}{$\begin{array}{l}\text { SMR E- } \\
\text { NFT, } \\
\text { inhibiting } \\
\text { theta and } \\
\text { high beta, } \\
\text { Z-score } \\
\text { based (13) }\end{array}$} & \multirow{3}{*}{$\begin{array}{l}\text { Indivi } \\
\text { dualiz } \\
\text { ed Z- } \\
\text { based } \\
\text { E-NFT } \\
(13)\end{array}$} & Beta and delta power & $\begin{array}{l}\text { Reduced after E-NFT sessions in } \\
\text { experimental group }\end{array}$ \\
\hline & & & $\begin{array}{l}\text { Total sleep time }(S) \\
\text { Sleep quality }(Q) \\
\text { Sleep efficiency (Q) } \\
\text { Insomnia severity (Q) } \\
\text { Quality of life (Q) }\end{array}$ & $\begin{array}{l}\text { Improved after E-NFT sessions, for both } \\
\text { groups combined }\end{array}$ \\
\hline & & & Wake after sleep onset (S) & $\begin{array}{l}\text { No significant change after E-NFT sessions in } \\
\text { both groups }\end{array}$ \\
\hline \multirow[t]{2}{*}{$\begin{array}{l}\text { Basiri et } \\
\text { al. (2017 } \\
[53])\end{array}$} & \multirow{2}{*}{$\begin{array}{l}\text { SMR E- } \\
\text { NFT, } \\
\text { inhibiting } \\
\text { theta and } \\
\text { high beta, } \\
\text { Z-score } \\
\text { based (20) }\end{array}$} & \multirow{2}{*}{$\begin{array}{l}\text { No } \\
\text { treat } \\
\text { ment } \\
\& \\
\text { CBT } \\
\text { (9) }\end{array}$} & Insomnia severity (Q) & $\begin{array}{l}\text { Improved after E-NFT sessions, as compared } \\
\text { to control and CBT group }\end{array}$ \\
\hline & & & Sleep quality (Q) & $\begin{array}{l}\text { Improved after E-NFT and CBT sessions, as } \\
\text { compared to control group, with higher } \\
\text { improvement for E-NFT group as compared } \\
\text { to CBT group }\end{array}$ \\
\hline \multirow{4}{*}{$\begin{array}{l}\text { Schabus } \\
\text { et al. } \\
(2014 \\
[54])\end{array}$} & \multirow[t]{4}{*}{$\begin{array}{l}\text { SMR E-NFT } \\
(10)\end{array}$} & \multirow{4}{*}{$\begin{array}{l}\text { Sham } \\
\text { E-NFT } \\
\text { (5) }\end{array}$} & SMR and high beta power & $\begin{array}{l}\text { Enhanced after E-NFT sessions in } \\
\text { experimental group }\end{array}$ \\
\hline & & & $\begin{array}{l}\text { Number awakenings (PSG) } \\
\text { SWS duration (PSG) }\end{array}$ & $\begin{array}{l}\text { Higher improvement after E-NFT sessions } \\
\text { for experimental as compared to control } \\
\text { condition }\end{array}$ \\
\hline & & & $\begin{array}{l}\text { Sleep quality (Q) } \\
\text { Physical quality of life (Q) }\end{array}$ & $\begin{array}{l}\text { Improved after E-NFT sessions, for both the } \\
\text { experimental as control condition }\end{array}$ \\
\hline & & & $\begin{array}{l}\text { Total sleep time (PSG+S) } \\
\text { Sleep onset latency (PSG+S) } \\
\text { Wake after sleep onset (PSG) } \\
\text { Sleep efficiency (PSG+S) } \\
\text { Time in bed (PSG) } \\
\text { Duration sleep stages other than } \\
\text { SWS (PSG) }\end{array}$ & $\begin{array}{l}\text { No significant change after E-NFT sessions in } \\
\text { both groups }\end{array}$ \\
\hline \multirow{3}{*}{$\begin{array}{l}\text { Schabus } \\
\text { et al. } \\
(2017 \\
[56])\end{array}$} & \multirow[t]{3}{*}{$\begin{array}{l}\text { SMR E-NFT } \\
(12)\end{array}$} & \multirow{3}{*}{$\begin{array}{l}\text { Sham } \\
\text { E-NFT } \\
(12)\end{array}$} & $\begin{array}{l}\text { EEG frequency bins, including } \\
\text { SMR power }\end{array}$ & $\begin{array}{l}\text { No significant change after E-NFT sessions in } \\
\text { both groups }\end{array}$ \\
\hline & & & $\begin{array}{l}\text { Sleep quality (Q) } \\
\text { Physical quality of life (Q) }\end{array}$ & $\begin{array}{l}\text { Improved after E-NFT sessions, for both the } \\
\text { experimental as control condition }\end{array}$ \\
\hline & & & $\begin{array}{l}\text { See variables of Schabus et al. } \\
\text { (2014) }\end{array}$ & $\begin{array}{l}\text { No significant change after E-NFT sessions in } \\
\text { both groups }\end{array}$ \\
\hline
\end{tabular}

Note: CBT $=$ cognitive behavioral therapy, EEG $=$ electroencephalography, EMG $=$ electromyography, E-NFT = EEG neurofeedbacktraining, $\mathrm{PSG}=$ polysomnography, $\mathrm{Q}=$ questionnaire, $\mathrm{S}=$ sleep diary, $\mathrm{SMR}=$ sensorimotor rhythm 


\section{CONCLUSION III}

The above reviewed literature indicate objective improvements in total sleep time, sleep onset latency, amount of awakening and SWS in insomnia patients after SMR E-NFT, and subjective improvements in insomnia severity, sleep efficiency, sleep quality and quality of life. Improvements of subjective sleep quality and physical quality of life in insomnia patients are probably rather due to unspecific E-NFT effects and not to the specific trained frequencies. In contrast, objective sleep improvements in the amount of awakening and SWS are found after successful enhancement of SMR after SMR ENFT. Other improvements might not become significant because high beta was not reduced during SMR E-NFT, which is normally seen, and this resulted in elevated high beta power. In addition, other waves such as high beta and delta might also be of importance for SMR E-NFT efficacy on sleep in insomnia patients. In most studies, unfortunately, the change in EEG after as compared to before treatment was not measured or noted. The improved objective sleep improvements, however, might also be related to unspecific effects such as social support.

\section{Neurofeedback effects on sleep in healthy individuals}

In this paragraph, the E-NFT effects on sleep in healthy individuals ae reviewed. First, tiredness during E-NFT and then the effect of sleep after E-NFT are discussed.

Furthermore, the above discussed SMR E-NFT technique as well as TAR E-NFT are emphasized. It should be again noted that theta waves are present when someone is sleepy, and alpha when someone is relaxed and alert [8]. While SMR E-NFT is characterized by visual or audiovisual feedback, in the studies below TAR E-NFT was provided by audio feedback only, and an eyes-closed state was required by the participants.

During E-NFT, tiredness was found to be increased after SMR and TBR E-NFT in healthy individuals [50]. Ten neurofeedback sessions were performed by 22 healthy students free of mental or neurological illness who did not take medication. In each session, SMR and beta E-NFT was given to the subjects in a counterbalanced order. High beta as well as theta power were reduced. Tiredness and tension were measured with questionnaires before and after each protocol. Tiredness was increased after the ENFT protocols, independent of the type of protocol. Tension was not changed after SMR or beta E-NFT.

Besides tiredness, also tension was found to be increased during E-NFT in healthy individuals after TAR or sham E-NFT, irrespective of type of E-NFT [57]. In this RCT, 18 healthy students were randomly assigned to an experimental $(n=9)$ and sham E-NFT group $(n=9)$. Five neurofeedback sessions were performed in total, and tiredness and tension was measured before and after each session. The experimental E-NFT consisted of TAR ENFT, while the feedback of a random session was played during sham E-NFT. While a significant increase was found within the sessions for the TAR as compared to the sham E-NFT group, no significant differences were found across sessions for both groups. Tiredness was increased and tension decreased after each E-NFT session, which was not dependent on experimental or sham E-NFT.

A positive effect of TAR E-NFT on sleep time and sleep architecture during the E-NFT was found in healthy individuals [58]. Forty mentally and somatically healthy young subjects were divided into a group receiving TAR E-NFT $(n=20)$ or sham E-NFT $(n=20)$. Sham E-NFT consisted of feedback of random training units from previous sessions. Ten neurofeedback sessions were completed by the subjects instructions to not fall asleep during the training were given. Objective sleep was measured during these sessions using PSG data. The TAR was significantly higher for both groups within the sessions, while changes in TAR across sessions were not noted. In total, 38 participants fell asleep at least once during the neurofeedback sessions. On average, all participants were asleep at one third of the neurofeedback sessions. While both groups showed the same amount of subjective sleepiness before the training, more stage 1 non-REM sleep and less time awake was observed during TAR ENFT as compared to sham E-NFT.

Also, a positive effect of TAR across TAR neurofeedback sessions on sleep onset latency was found in healthy individuals [59]. Thirty-five young healthy participants that did not use medication listened to relaxation music while TAR E-NFT was provided in the experimental condition or no feedback was given as a control condition. After eight sessions, TAR enhancement was found in the TAR group as compared to control group. In addition, successful TAR enhancement was associated with the transition from awake to sleep states, which was verified with measures of thumb and index finger pressure. Based on these results, it was concluded that the sleep onset 
latency based on the TAR was significantly reduced after TAR E-NFT as compared to the control group.

No effects after E-NFTs were found in healthy individuals wherein SMR was not successfully trained [60]. In this study, sleep was examined in 11 students free of mental or physical illnesses, mood disorders and sleep disturbances, that received sigma E-NFT and sham E-NFT in a counterbalanced design. During sigma E-NFT, the SMR was trained, as well as the upper alpha wave frequency band, which is related to optimizing cognitive performance [8]. During sham E-NFT, random feedback was given. Before and after four training blocks of E-NFT, sleep architecture was measured, which depicts the amount of time spend in each sleep stage, with PSG. Furthermore, subjective sleep such as the total sleep time and wake after sleep onset was measured with sleep diaries. No significant changes were found on objective and subjective sleep after sigma or sham E-NFT. In addition, the four $10 \mathrm{~min}$ blocks of E-NFT failed to significantly enhance SMR or other frequency bands.

Results of a single-blind RCT indicated a positive effect of successfully trained SMR after SMR E-NFT on sleep onset latency in healthy individuals [61]. Twenty-seven young subjects free of mood disorders, intelligence or learning problems and sleep disturbances were randomly assigned to a group receiving ten sessions of SMR E-NFT $(n=16)$ or sham E-NFT $(n=10)$. Sham E-NFT consisted of random frequency bins trained in each session. Objective sleep measures were performed using polygraphic sleep recordings during a 90 min nap before and after the ten neurofeedback sessions. In contrast to Berner et al. [60], a significant SMR enhancement and a shorter sleep onset latency was found after the neurofeedback sessions in the SMR E-NFT group only. Also, a trend of increased total sleep time and more SWS was found after SMR and not after sham E-NFT.

\section{CONCLUSION IV}

Neurofeedback seems to increase tiredness and decrease tension in healthy individuals during the application of E-NFT. In the case of eyeclosed TAR neurofeedback sessions, but possibly also in other experimental neurofeedback sessions, this effect is rather due to unspecific effects because it is also found in the nonexperimental E-NFT condition. In addition, successful training of TAR across neurofeedback sessions shortened the sleep onset latency and seemed also to decrease awake times and to increase the amount stage 1 nonREM sleep observed during the training. A shortened sleep onset latency is also observed after SMR neurofeedback sessions, but only when SMR is successfully trained across these neurofeedback sessions. Training of TAR and SMR across neurofeedback sessions seems to be more successful when more training sessions are applied.

\section{DISCUSSION}

The aim of this literature review was to examine the efficacy of EEG-NFT on attention and sleep in individuals with ADHD or insomnia disorders as well as in individuals without these problems . Meta-analyses provide evidence that standard E-NFT protocols which are TBR, SMR and SCP E-NFT, have a positive longlasting effect on the inattention and HI symptoms in children with ADHD. In healthy children and young adults, TBR seems to improve sustained and selective attention, SMR sustained attention, TAR E-NFT impulsivity and theta E-NFT attention control. There also seems to be a positive effect of SMR and TAR E-NFT on the objective and subjective sleep onset latency, and an unspecific E-NFT effect on tiredness and tension in healthy individuals. Objective and subjective sleep improvements are found in insomnia patients after SMR E-NFT as well, with it being argued that the subjective sleep improvements likely depend on unspecific E-NFT effects. Also, reduced high beta and delta power after SMR E-NFT might be important for SMR E-NFT efficacy in sleep.

Taken together, EEG E-NFT seems to have a positive effect on attention and impulsivity in subjects with ADHD and healthy young people. In addition, sleep improvements in insomnia patients might rely on the ENFT protocol used, the amount of impaired sleep quality of the insomnia patient or be related to unspecific E-NFT effects. Sleep improvements in healthy people can also be 
found after E-NFT, and direct unspecific E-NFT effects on tiredness.

For attention, standard E-NFT protocols seem to be important for the efficacy in healthy young people and children with ADHD. In healthy young people, enhanced SMR and beta power through SMR and TBR E-NFT have a positive effect on attention, while enhanced theta power through TAR and theta E-NFT have a positive effect on impulsivity and attention control. In children with ADHD, a positive effect of SMR, TBR and SCP ENFT is found on both inattention and HI symptoms, while it might be expected that only attentional symptoms might be reduced because of enhanced SMR and beta but not necessary theta power. However, differential effects of E-NFT in ADHD patients have been found as well. First of all, the long-lasting effects of E-NFT after the end of the treatment was higher for the inattention symptoms. Also, only the effect on HI symptoms was dependent on the amount of blinding in the studies. In addition, efficacy of E-NFT to reduce HI symptoms was no longer found when blinded raters were considered. It is therefore possible that in children with ADHD, E-NFT protocols aimed to enhance SMR and beta power can improve the inattentive symptoms, and those aimed to enhance theta power to improve HI symptoms, as in line with the findings in healthy young people. However, that HI is not always reduced based on blinded raters can be accounted to an underestimation of these symptoms by these raters. Therefore, SMR and TBR E-NFT might reduce inattention in healthy young people and both inattention and $\mathrm{HI}$ in children with ADHD.

For sleep, standard and Z-score based SMR E-NFT protocols were used. In healthy individuals, more tiredness and less tension are found that are not explained by the trained frequency but is rather due to the E-NFT protocol wherein the subjects had to close their eyes and were instructed to relax. Also in SMR and TBR E-NFT that requires an active state, increased tiredness was found after each E-NFT session. Unspecific training effects in SMR and TBR E-NFT would rather include concentration, motivation and expectation. As there was no specific control group, tiredness as a results of SMR and TBR E-NFT is not necessarily indicated because of unspecific training effects. However, also in insomnia patients, subjective sleep improvements were assumed to be improved by unspecific E-NFT effects of SMR E-NFT, because they did not depend on the trained frequency during E-NFT. This is not necessarily true for objective sleep, which was increased after enhanced SMR and was unaltered when SMR enhancement was not successful. In addition, healthy individuals also showed a decreased sleep onset latency after successful SMR enhancement after SMR E-NFT. Thus, it is speculated that in both healthy individuals and insomnia patients, nonexperimental relaxation and possibly also activating $\mathbf{E}$ NFT procedures can have a positive effect on tiredness and give rise to some subjective sleep improvements. However, SMR enhancement after SMR E-NFT can improve objective sleep in healthy individuals and therefore improve the insomnia symptomatology even more in patients, therefore making it an efficacious treatment for insomnia.

The efficacy of standard E-NFT protocols for the treatment of ADHD is in line with previous studies that found improved inattention and HI symptoms in children with ADHD after TBR and SCP E-NFT [16, 31, 32] and after SMR and TBR E-NFT [20]. These positive effects on attention after TBR and SMR E-NFT is also found in healthy young people [46-48]. Furthermore, faster reaction time on a sustained attention task was found in healthy young adults after TBR E-NFT as compared to the control group [47]. This is also in line with earlier research, that found faster reaction times and better scores on a sustained attention task after beta E-NFT [18]. One already mentioned different effect between children with ADHD and young people after E-NFT is that concerning impulsivity. Lower impulsivity on a sustained attention task was found after SMR and TBR E-NFT in children with ADHD [20], but this was not found in healthy young adults [47, 48]. This reduction in impulsivity on the same task used by Fuchs et al. [20] was found in school children without ADHD after TAR E-NFT [49]. It is possible that different tasks must be used in healthy individuals to find an effect on impulsivity. Of course, it is also possible that only in children with ADHD the standard protocols have an effect on impulsivity besides attention as well. In healthy individuals, reduced impulsive responses might then be realized rather by TAR relaxation E-NFT [49].

The effects on sleep in healthy people were not investigated in the previous century. In an epilepsy patient, however, reduced sleep onset latency was found after SMR E-NFT [23], but only when the SMR was successfully enhanced. Improved sleep onset latency after SMR E-NFT was also found in a RCT with healthy individuals [61], but again this was not true when the SMR was not successfully trained [60]. The positive correlation of baseline tension of insomnia patients with the sleep improvements after theta E-NFT [26], can be explained by the observed effect of theta E-NFT in healthy individuals on reducing tension and increasing tiredness after each $\mathrm{E}$ NFT session [57], increasing time asleep during the sessions [58] and reducing sleep onset latency across the sessions [59]. The reduced sleep onset latency after theta E-NFT is implied and not an objective measure [59]. In insomnia patients, however, no change in sleep onset latency was found after theta E-NFT, while it did improve 
the number of awakenings [26]. After SMR E-NFT, improvements were found in insomnia patients on total sleep time [2, 27] and sleep onset latency [2] based on sleep diaries. This was also found in the study of Hauri et al. [26]. Objective sleep improvements on total sleep time after SMR E-NFT were found in insomnia patients as well for total sleep time [2] and the amount of SWS and awakenings [54]. Another study did not find any objective sleep improvements after SMR E-NFT [56]. No objective sleep improvements in insomnia patients after SMR ENFT was also found in the previous studies [25, 26]. In addition, the negative correlation between the baseline tension of the insomnia patients and the sleep improvements that were found after SMR E-NFT [25, 26], could not be found in later studies $[2,56]$. One difference is that in the studies of Hauri [25] and Hauri et al. [26], the subjects are psychophysiological insomnia patients that experience cognitive and physical arousal before sleep, such as tension, while later studies were not specifically focused on this subtype of insomnia. In addition, the baseline tension levels of the insomnia patients were not significantly higher than of healthy controls in the study that did experience sleep improvements after SMR ENFT [2]. This is in line with the association between less tense insomnia patients and better outcomes after SMR ENFT [25, 26]. Other studies did not measure or note these baseline tension levels of the subjects. Also, SMR and other EEG changes after SMR E-NFT were not checked in the study of Hauri et al. [26], and it is argued earlier in this discussion that EEG changes other than SMR might be important for the SMR E-NFT efficacy in insomnia patients.

Although the exact working mechanisms of E-NFT are not yet clear, some suggestions are made based on this review. Overall disturbances in TBR in children with ADHD are normalized by TBR E-NFT [12, 13], and provide an increased attentional over sleepy states [8]. Besides an increase in overall attention, it provides improvement in selective attention, also in healthy individuals. Selective attention is thought to be important for inhibitory control which is related to impulsivity [62], which might explain why reduced impulsivity after TBR ENFT is found in children showing problems with impulsivity due to ADHD. SMR E-NFT rather decreases motor hyperactivity and impulsivity [21, 22]. Therefore, less motor information has to be processed in sensorimotor areas, and more information related to executive functions such as attention is provided [63]. This may give rise to improved sustained attention in children with ADHD, but not in healthy children that do not exhibit this hyperactivity. Increased motor inhibition after SMR E-NFT is also beneficial for sleep in healthy individuals and insomnia patients. SMR E-NFT also reduces high beta, which is elevated in insomnia patients and is associated with more negative perception of sleep quality [64]. Excessive low frequency delta and theta waves in insomnia patients might be related to being exhausted by the disease [27], and reduced power might improve mental or physical state as well. When increased tension is found in the insomnia patients that is related to negative thoughts about sleep [24], theta E-NFT might be more appropriate. Theta E-NFT seems to decrease tension, but may also provide a more rapid transition into sleep, also in healthy individuals. Decreased tension and a more relaxed state after theta E-NFT might also increase the cognitive load in healthy individuals that is needed for increased inhibitory control.

Unfortunately, the amount of studies on E-NFT with a good methodology, which are double-blind RCTs, are also in recent years rather scarce. Moreover, only one doubleblind RCT has been performed on E-NFT effects on sleep in insomnia patients. It becomes clear, however, that especially in EEG E-NFT studies, a good methodological design is harder to achieve and also debatable. First, for a double-blind design a sham E-NFT condition is needed. In this sham E-NFT condition, the operant conditioning principle must still be respected [36]. This means that random frequency bins must be trained, without it providing significantly enhanced EEG power in certain frequency waves. Studies have shown, however, that this is possible. It is also mentioned that it is better to not use sham E-NFT as a control condition, because the so-called unspecific E-NFT effects such as motivation and control beliefs are characteristic for E-NFT and its efficacy [65]. It is a scientific consensus, however, for a treatment to be efficacious if it shows improved effects relative to a sham condition. This seems to be the case for ADHD, but unfortunately still today needs to be investigated for insomnia. Also, sleep improvements after E-NFT in patients other than with insomnia and attention improvements after E-NFT in patients other than ADHD must be investigated. In addition, after TBR E-NFT improvements in sustained attention and response inhibition were also found in children with autism spectrum disorder [66], and improvements in sustained, selective and inhibitory control in children with dyslexia [67]. In migraine patients, more than the half of the patients showed improved sleep after Z-score based ENFT in patients with migraine [68]. Furthermore, after SMR and TBR E-NFT, improvements in sleep quality were found in adults with ADHD [11] and less sleep problems in children with ADHD [69]. Although the study of van Dongen-Boomsma et al. [69] was a doubleblind RCT, the applied experimental E-NFT protocol is criticized as it probably prevented the desired frequency bands to be learned by the participants [36]. The successful training of the experimental waves after E-NFT is thought to be important for its effect on sleep as well as 
attention in healthy individuals as well as in ADHD and in insomnia patients. The EEG changes after E-NFT are, unfortunately, often not recorded. Future research should also focus on the effects of E-NFT on attention in patients other than those with ADHD, and the E-NFT effects on sleep in insomnia patients as well as in patients with other disorders. Furthermore, it should be investigated why some people do and some people do not successfully train specific brainwaves following E-NFT, to increase the effects on behavior in healthy people or to more successfully reduce symptoms in patients.
1. Strehl, U., What learning theories can teach us in designing neurofeedback treatments. Frontiers in human neuroscience, 2014. 8: p. 894.

2. Cortoos, A., E. De Valck, M. Arns, M.H. Breteler and R. Cluydts, An exploratory study on the effects of tele-neurofeedback and telebiofeedback on objective and subjective sleep in patients with primary insomnia. Applied psychophysiology and biofeedback, 2010. 35(2): p. 125-134.

3. Engelbregt, H.J., D. Keeser, L. van Eijk, E.M. Suiker, D. Eichhorn, S. Karch, J.B. Deijen and O. Pogarell, Short and long-term effects of shamcontrolled prefrontal EEGneurofeedback training in healthy subjects. Clin Neurophysiol, 2016. 127(4): p. 1931-7 DOI: 10.1016/j.clinph.2016.01.004.

4. $\quad$ Engelbregt, H.J., G. Kok, R. Vis, D. Keeser and J.B. Deijen, Instrumentele conditionering van frontaalkwabactiviteit bij gezonde jongvolwassenen. Tijdschrift voor Neuropsychologie, 2010. 5(1): p. 16-26.

5. Keizer, A.W., R.S. Verment and B. Hommel, Enhancing cognitive control through neurofeedback: A role of gamma-band activity in managing episodic retrieval. Neuroimage, 2010. 49(4): p. 3404-3413.

6. Zoefel, B., R.J. Huster and C.S. Herrmann, Neurofeedback training of the upper alpha frequency band in EEG improves cognitive performance. Neuroimage, 2011. 54(2): p. 1427 1431.

7. Eijk, L.v., S. Zwijsen, D. Keeser, J. Oosterman, O. Pogarell and H. Engelbregt, EEG-neurofeedback training and quality of life of institutionalized elderly women: A pilot study. 2017.

\section{REFERENCES}

8. Marzbani, H., H.R. Marateb and M. Mansourian, Neurofeedback: a comprehensive review on system design, methodology and clinical applications. Basic and clinical neuroscience, 2016. 7(2): p. 143.

9. Association, A.P. and A.P. Association, Diagnostic and statistical manual of mental disorders: DSM-5. Arlington, VA, 2013.

10. Enriquez-Geppert, S., D. Smit, M.G. Pimenta and M. Arns, Neurofeedback as a treatment intervention in $A D H D$ : Current evidence and practice. Current psychiatry reports, 2019. 21(6): p. 1-7.

11. Arns, M. and J.L. Kenemans, Neurofeedback in ADHD and insomnia: vigilance stabilization through sleep spindles and circadian networks. Neuroscience \& Biobehavioral Reviews, 2014. 44: p. 183-194.

12. Barry, R.J., A.R. Clarke, S.J. Johnstone, R. McCarthy and M. Selikowitz, Electroencephalogram $\theta \beta$ ratio and arousal in attention-deficit/hyperactivity disorder: Evidence of independent processes. Biological psychiatry, 2009. 66(4): p. 398-401.

13. Lazzaro, I., E. Gordon, W. Li, C. Lim, M. Plahn, S. Whitmont, S. Clarke, R. Barry, A. Dosen and R. Meares, Simultaneous EEG and EDA measures in adolescent attention deficit hyperactivity disorder. International Journal of Psychophysiology, 1999. 34(2): p. 123-134.

14. Birbaumer, N., T. Elbert, A.G. Canavan and B. Rockstroh, Slow potentials of the cerebral cortex and behavior. Physiological reviews, 1990. 70(1): p. 1-41.

15. Rockstroh, B., T. Elbert, N. Birbaumer and W. Lutzenberger, Biofeedbackproduced hemispheric asymmetry of slow cortical potentials and its 
behavioural effects. International

Journal of Psychophysiology, 1990.

9(2): p. 151-165.

16. Gevensleben, H., B. Holl, B. Albrecht, C. Vogel, D. Schlamp, O. Kratz, P. Studer, A. Rothenberger, G.H. Moll and H. Heinrich, Is neurofeedback an efficacious treatment for ADHD? A randomised controlled clinical trial. Journal of Child Psychology and Psychiatry, 2009. 50(7): p. 780-789.

17. Drechsler, R., M. Straub, M. Doehnert, H. Heinrich, H.-C. Steinhausen and D. Brandeis, Controlled evaluation of a neurofeedback training of slow cortical potentials in children with attention deficit/hyperactivity disorder (ADHD). Behavioral and Brain Functions, 2007. 3(1): p. 35.

18. Rasey, H., J.F. Lubar, A. McIntyre, A. Zoffuto and P.L. Abbott, $E E G$ biofeedback for the enhancement of attentional processing in normal college students. Journal of Neurotherapy, 1995. 1(3): p. 15-21.

19. Darian-Smith, C., I. Darian-Smith and S.S. Cheema, Thalamic projections to sensorimotor cortex in the macaque monkey: use of multiple retrograde fluorescent tracers. Journal of Comparative Neurology, 1990. 299(1): p. 17-46.

20. Fuchs, T., N. Birbaumer, W. Lutzenberger, J.H. Gruzelier and J. Kaiser, Neurofeedback treatment for attention-deficit/hyperactivity disorder in children: a comparison with methylphenidate. Applied psychophysiology and biofeedback, 2003. 28(1): p. 1-12.

21. Lubar, J.F. and M.N. Shouse, EEG and behavioral changes in a hyperkinetic child concurrent with training of the sensorimotor rhythm (SMR).

Biofeedback and Self-regulation, 1976. 1(3): p. 293-306.

22. Shouse, M. and J. Lubar, Operant conditioning of EEG rhythms and ritalin in the treatment of hyperkinesis. Biofeedback and Self-regulation, 1979. 4(4): p. 299-312.

23. Sterman, M. and L. Friar, Suppression of seizures in an epileptic following sensorimotor EEG feedback training.
Electroencephalography and clinical neurophysiology, 1972. 33(1): p. 89-95.

24. Perlis, M. and P. Gehrman, Types of insomnia. Encyclopedia of Sleep, 2013: p. 199-202.

25. Hauri, P., Treating psychophysiologic insomnia with biofeedback. Archives of General Psychiatry, 1981. 38(7): p. 752758.

26. Hauri, P.J., L. Percy, C. Hellekson, E. Hartmann and D. Russ, The treatment of psychophysiologic insomnia with biofeedback: A replication study. Biofeedback and Self-regulation, 1982. 7(2): p. 223-235.

27. Hammer, B.U., A.P. Colbert, K.A. Brown and E.C. Ilioi, Neurofeedback for insomnia: A pilot study of Z-score SMR and individualized protocols. Applied psychophysiology and biofeedback, 2011. 36(4): p. 251-264.

28. Collura, T.F., R.W. Thatcher, M. Smith, W. Lambos and C. Stark, EEG biofeedback training using live Z-scores and a normative database. Introduction to quantitative EEG and neurofeedback, 2009: p. 103-141.

29. Arns, M., S. de Ridder, U. Strehl, M. Breteler and A. Coenen, Efficacy of neurofeedback treatment in ADHD: the effects on inattention, impulsivity and hyperactivity: a meta-analysis. Clin EEG Neurosci, 2009. 40(3): p. 180-9 DOI: 10.1177/155005940904000311.

30. Lansbergen, M.M., M. van DongenBoomsma, J.K. Buitelaar and D. SlaatsWillemse, $A D H D$ and $E E G$ neurofeedback: a double-blind randomized placebo-controlled feasibility study. Journal of neural transmission, 2011. 118(2): p. 275-284.

31. Drechsler, R., M. Straub, M. Doehnert, H. Heinrich, H.-C. Steinhausen and D. Brandeis, Controlled evaluation of a neurofeedback training of slow cortical potentials in children with attention deficit/hyperactivity disorder (ADHD). Behavioral and brain functions, 2007. 3(1): p. 1-13.

32. Heinrich, H., H. Gevensleben, F.J. Freisleder, G.H. Moll and A. Rothenberger, Training of slow cortical potentials in attentiondeficit/hyperactivity disorder: evidence for positive behavioral and 
neurophysiological effects. Biological psychiatry, 2004. 55(7): p. 772-775.

33. Kemper, A.R., G.R. Maslow, S. Hill, B. Namdari, N.M.A. LaPointe, A.P.

Goode, R.R. Coeytaux, D. Befus, A.S. Kosinski and S.E. Bowen, Attention deficit hyperactivity disorder: Diagnosis and treatment in children and adolescents. 2018.

34. Wolraich, M., L. Brown, R. Brown, G. DuPaul, M. Earls, H. Feldman, T.

Ganiats, B. Kaplanek, B. Meyer and J.

Perrin, ADHD: clinical practice guideline for the diagnosis, evaluation, and treatment of attentiondeficit/hyperactivity disorder in children and adolescents. Pediatrics, 2011. 128(5): p. 1007-1022.

35. Sonuga-Barke, E.J., D. Brandeis, S. Cortese, D. Daley, M. Ferrin, M.

Holtmann, J. Stevenson, M.

Danckaerts, S. Van der Oord and M.

Döpfner, Nonpharmacological interventions for ADHD: systematic review and meta-analyses of randomized controlled trials of dietary and psychological treatments.

American Journal of Psychiatry, 2013. 170(3): p. 275-289.

36. Arns, M., H. Heinrich and U. Strehl, Evaluation of neurofeedback in ADHD: the long and winding road. Biological psychology, 2014. 95: p. 108115.

37. Bussalb, A., M. Congedo, Q. Barthélemy, D. Ojeda, E. Acquaviva, R. Delorme and L. Mayaud, Clinical and experimental factors influencing the efficacy of neurofeedback in ADHD: a meta-analysis. Frontiers in psychiatry, 2019. 10: p. 35.

38. Riesco-Matías, P., J.R. Yela-Bernabé, A. Crego and E. Sánchez-Zaballos, What do meta-analyses have to say about the efficacy of neurofeedback applied to children with ADHD? Review of previous meta-analyses and a new meta-analysis. Journal of attention disorders, 2021. 25(4): p. 473-485.

39. Cortese, S., M. Ferrin, D. Brandeis, M. Holtmann, P. Aggensteiner, D. Daley, P. Santosh, E. Simonoff, J. Stevenson and A. Stringaris, Neurofeedback for attention-deficit/hyperactivity disorder: meta-analysis of clinical and neuropsychological outcomes from randomized controlled trials. Journal of the American Academy of Child \& Adolescent Psychiatry, 2016. 55(6): p. 444-455.

40. Micoulaud-Franchi, J.-A., P.A. Geoffroy, G. Fond, R. Lopez, S. Bioulac and P. Philip, EEG neurofeedback treatments in children with ADHD: an updated meta-analysis of randomized controlled trials. Frontiers in human neuroscience, 2014. 8: p. 906.

41. Van Doren, J., M. Arns, H. Heinrich, M.A. Vollebregt, U. Strehl and S.K.

Loo, Sustained effects of neurofeedback in ADHD: a systematic review and meta-analysis. European child \& adolescent psychiatry, 2019. 28(3): p. 293-305.

42. Leins, U., G. Goth, T. Hinterberger, C. Klinger, N. Rumpf and U. Strehl, Neurofeedback for children with ADHD: a comparison of SCP and Theta/Beta protocols. Applied psychophysiology and biofeedback, 2007. 32(2): p. 73-88.

43. Mirsky, A.F., B.J. Anthony, C.C. Duncan, M.B. Ahearn and S.G. Kellam, Analysis of the elements of attention: A neuropsychological approach. Neuropsychology review, 1991. 2(2): p. 109-145.

44. Holmboe, K. and M.H. Johnson, Educating executive attention. Proceedings of the National Academy of Sciences, 2005. 102(41): p. 1447914480.

45. San Luis, C., A. López de la Llave and M.C. Pérez-Llantada, Training to improve selective attention in children using neurofeedback through play. Revista de Psicopatología y Psicología Clínica, 2013. 18(3): p. 209-216.

46. Lutsyuk, N., E. Éismont and V. Pavlenko, Modulation of attention in healthy children using a course of EEGfeedback sessions. Neurophysiology, 2006. 38(5-6): p. 389-395.

47. Egner, T. and J.H. Gruzelier, $E E G$ biofeedback of low beta band components: frequency-specific effects on variables of attention and eventrelated brain potentials. Clinical 
neurophysiology, 2004. 115(1): p. 131139.

48. Vernon, D., T. Egner, N. Cooper, T. Compton, C. Neilands, A. Sheri and J. Gruzelier, The effect of training distinct neurofeedback protocols on aspects of cognitive performance. International journal of psychophysiology, 2003. 47(1): p. 75-85.

49. Gruzelier, J.H., M. Foks, T. Steffert, M.-L. Chen and T. Ros, Beneficial outcome from EEG-neurofeedback on creative music performance, attention and well-being in school children. Biological psychology, 2014. 95: p. 8695.

50. Gruzelier, J.H., Differential effects on mood of 12-15 (SMR) and 15-18 (beta1) Hz neurofeedback.

International Journal of

Psychophysiology, 2014. 93(1): p. 112115.

51. Wang, J.-R. and S. Hsieh, Neurofeedback training improves attention and working memory performance. Clinical

Neurophysiology, 2013. 124(12): p. 2406-2420 DOI:

http://dx.doi.org/10.1016/j.clinph.2013. 05.020.

52. $\quad$ Engelbregt, H.J., D. Keeser, V.H. Promes, S. Verhagen-Schouten and J.B. Deijen, In-vivo EEG changes during a panic attack in a patient with specific phobia. Journal of Medical Cases, 2012. 3(1): p. 34-38 DOI: 10.4021/jmc403e.

53. Basiri, N., Z. Khayyer, H. Hadianfard and A. Ghaderi, Comparison of the effectiveness of cognitive behavioral therapy and neurofeedback: reducing insomnia symptoms. Global J Health Sci, 2017. 9(7).

54. Schabus, M., D.P. Heib, J. Lechinger, H. Griessenberger, W. Klimesch, A. Pawlizki, A.B. Kunz, B.M. Sterman and K. Hoedlmoser, Enhancing sleep quality and memory in insomnia using instrumental sensorimotor rhythm conditioning. Biological psychology, 2014. 95: p. 126-134.

55. Edinger, J.D., M.H. Bonnet, R.R. Bootzin, K. Doghramji, C.M. Dorsey, C.A. Espie, A.O. Jamieson, W.V. McCall, C.M. Morin and E.J.
Stepanski, Derivation of research diagnostic criteria for insomnia: report of an American Academy of Sleep Medicine Work Group. Sleep, 2004. 27(8): p. 1567-1596.

56. Schabus, M., H. Griessenberger, M.-T. Gnjezda, D.P. Heib, M. Wislowska and K. Hoedlmoser, Better than sham? A double-blind placebo-controlled neurofeedback study in primary insomnia. Brain, 2017. 140(4): p. 10411052.

57. Egner, T., E. Strawson and J.H. Gruzelier, EEG signature and phenomenology of alpha/theta neurofeedback training versus mock feedback. Applied psychophysiology and biofeedback, 2002. 27(4): p. 261270.

58. Schütze, M.D. and K. Junghanns, The difficulty of staying awake during alpha/theta neurofeedback training. Applied psychophysiology and biofeedback, 2015. 40(2): p. 85-94.

59. Vafaei, E., M.S.K. Hosseini and S.M.M. Shushtarian. Developing a fuzzy theta alpha neurofeedback for treatment of pre-sleep disorders. in 2015 22nd Iranian Conference on Biomedical Engineering (ICBME). 2015. IEEE.

60. Berner, I., M. Schabus, T. Wienerroither and W. Klimesch, The significance of sigma neurofeedback training on sleep spindles and aspects of declarative memory. Applied psychophysiology and biofeedback, 2006. 31(2): p. 97.

61. Hoedlmoser, K., T. Pecherstorfer, G. Gruber, P. Anderer, M. Doppelmayr, W. Klimesch and M. Schabus, Instrumental conditioning of human sensorimotor rhythm $(12-15 \mathrm{~Hz})$ and its impact on sleep as well as declarative learning. Sleep, 2008. 31(10): p. 14011408.

62. Lackner, C.L., D.L. Santesso, J. Dywan, T.J. Wade and S.J. Segalowitz, Electrocortical indices of selective attention predict adolescent executive functioning. Biological psychology, 2013. 93(2): p. 325-333.

63. Kober, S.E., M. Witte, M. Stangl, A. Väljamäe, C. Neuper and G. Wood, Shutting down sensorimotor 
interference unblocks the networks for stimulus processing: An SMR

neurofeedback training study. Clinical Neurophysiology, 2015. 126(1): p. 8295.

64. Perlis, M.L., M.T. Smith, P.J. Andrews, H. Orff and D.E. Giles, Beta/Gamma EEG activity in patients with primary and secondary insomnia and good sleeper controls. Sleep, 2001. 24(1): p. 110-117.

65. Witte, M., S.E. Kober and G. Wood, Noisy but not placebo: defining metrics for effects of neurofeedback. Brain, 2018.

66. Kouijzer, M.E., J.M. de Moor, B.J. Gerrits, J.K. Buitelaar and H.T. van Schie, Long-term effects of neurofeedback treatment in autism. Research in Autism Spectrum Disorders, 2009. 3(2): p. 496-501.
67. Au, A., G.S. Ho, E.W. Choi, P. Leung, M.M. Waye, K. Kang and K.-y. Au, Does it help to train attention in dyslexic children: pilot case studies with a ten-session neurofeedback program. International journal on disability and human development, 2014. 13(1): p. 45-54.

68. Stokes, D.A. and M.S. Lappin, Neurofeedback and biofeedback with 37 migraineurs: a clinical outcome study. Behavioral and Brain Functions, 2010. 6(1): p. 1-10.

69. van Dongen-Boomsma, M., M.A. Vollebregt, D. Slaats-Willemse and J.K. Buitelaar, A randomized placebocontrolled trial of electroencephalographic (EEG) neurofeedback in children with attention-deficit/hyperactivity disorder. The Journal of clinical psychiatry, 2013. 74(8): p. 0-0. 


\section{ABBREVIATIONS}

ADHD $=$ attention deficit hyperactivity disorder

Beta E-NFT = beta power enhancing E-NFT

$\mathrm{CBT}=$ cognitive behavioral therapy

$\mathrm{CPT}=$ continuous performance task

DSM = diagnostic and statistical manual of mental disorders

EEG = electroencephalography

EMG = electromyography

E-NFT = electroencephalography neurofeedbacktraining

$\mathrm{HI}=$ hyperactivity/impulsivity

$\mathrm{NF}=$ neurofeedback

$\mathrm{PB}=$ probably blinded

PSG = polysomnography

$\mathrm{PU}=$ probably unblinded

$\mathrm{q}=$ questionnaire

$\mathrm{RCT}=$ randomized controlled trial

REM = rapid eye movement

$\mathrm{S}=$ sleep diary

SCP $=$ slow cortical potential

SMR = sensorimotor rhythm

$\mathrm{SWS}=$ slow wave sleep

$\mathrm{TAR}=$ theta/alpha ratio

$\mathrm{TBR}=$ theta/beta ratio

Theta E-NFT = theta power enhancing E-NFT 